\title{
Desarrollo del pensamiento contable: ¿hacer o saber contable?*
}

Martha Acosta*

Recibido: 14 de abril de 2012

Aprobado: 17 de junio de 2012

\section{Acosta, M. (2012). Desarrollo del pensamiento contable: ¿̨hacer o saber contable? Activos, 19, 129-155.}

\section{JEL Z00}

\section{Resumen}

El trabajo desarrolla una reflexión en torno a la relación entre "hacer" y "saber" contable, el cual ha sido un elemento de tensión constante en el ámbito de la academia contable en Colombia.

El documento analiza la importancia de romper el imaginario según el cual el concepto de contabilidad es de orden técnico e instrumental. El presente artículo presenta las corrientes de pensamiento contable más representativas y señala cómo todo este saber contable se deriva principalmente de serios procesos de investigación, que han puesto su mirada en las problemáticas contables de orden práctico que se han presentado en el mundo económico y organizacional a lo largo de la historia, y cómo el concepto de contabilidad y su cuerpo teórico ha evolucionado, ha presentado

\footnotetext{
* Artículo derivado del proyecto de Investigación "Observatorio Colombiano de Educación Contable” (tipo III), financiado por la Universidad Santo Tomás.

${ }^{* *}$ Docente de la Facultad de Contaduría Pública, Universidad Santo Tomás. Correo electrónico: marthaacosta@usantotomas.edu.co
} 
nuevas soluciones de medición, valuación, control, entre otras. Lo anterior pone en evidencia que el hacer y el saber contable son dos campos de conocimiento que se han desarrollado a la par.

\title{
Palabras clave:
}

doctrinas contables, paradigmas contables, programas de investigación, tradiciones de investigación, investigación contable.

\section{Acosta, M. (2012). Development of accounting thinking: accoun- ting doing or knowing? Activos, 19, 129-155.}

\begin{abstract}
The work develops a reflection on the relationship between accounting "doing" and "knowing", which has been a constant tension issue in the field of accounting academia in Colombia.

The paper discusses the importance of breaking the imagery whereby the accounting concept is technical and instrumental. This article presents the most representative schools of accounting thought and shows how all this knowledge is mainly derived from serious accounting research processes, which have set eyes on practical accounting problems that have occurred in the economic and organizational world throughout history, and how the accounting concept and theoretical framework has evolved, has presented new solutions for measurement, valuation, control among others. The above shows that accounting doing and knowing are two knowledge fields that have developed alongside.
\end{abstract}

\section{Keywords:}

accounting doctrines, accounting paradigms, research programs, research traditions, accounting research. 


\section{Acosta, M. (2012). Développement de la pensée comptable: faire ou savoir comptable? Activos, 19, 129-155.}

\section{Résumé}

Ce travail est une réflexion sur la relation entre " faire " et "savoir " comptable, élément de tension constante dans le domaine de l'académie comptable en Colombie.

Le document analyse l'importance de rompre avec l'imaginaire selon lequel le concept de comptabilité est de l'ordre technique et instrumental. Cet article présente les courants de pensée comptable les plus représentatives et signale comment tout ce savoir comptable ressort principalement de recherches sérieuses qui ont visé sur les problématiques comptables de l'ordre pratique qui se sont présentées dans le monde économique et organisationnel tout au long de l'histoire, et comment le concept de comptabilité et son corpus théorique a évolué et a présenté de nouvelles solutions de mesure, évaluation, contrôle, entre autres. Ceci met en évidence que le faire et le savoir comptable sont des champs de connaissance qui se sont développés au même temps.

\section{Mots-clés:}

doctrines comptables, paradigmes comptables, programmes de recherche, traditions de recherche, recherche comptable.

\section{Introducción}

En la primera parte del presente artículo se presenta la evolución del pensamiento contable abordado por los principales documentos y publicaciones contables desde el siglo XV hasta nuestros días. Los aportes teóricos allí evidenciados se han convertido en doctrinas, paradigmas o tendencias, las cuales han dado respuestas teóricas y prácticas a los fenómenos económicos 
y necesidades organizacionales que se han originado en cada momento histórico.

Muchos de los principales aportes a las disciplina se han creado en estudios de algunos interesados y conocedores del tema; sin embargo, desde el siglo pasado, varios aportes al cuerpo teórico contable se han derivado de serios trabajos de investigación, tema que se desarrolla en la segunda parte del documento. Estos grupos de investigación se han conformados especialmente en el seno de la academia y han estado preocupados por afianzar el carácter científico de la contabilidad; además, han propuesto un cuerpo teórico contable a través de nuevas teorías, doctrina, principios, marcos conceptuales, regulación y nuevas propuestas de lo que debe ser la contabilidad y su valor agregado para las organizaciones, la economía y la sociedad en general.

A partir de estas investigaciones y sus orígenes de orden práctico, en la última parte del artículo se presenta una polémica aún no resuelta acerca de la profesión, centrada especialmente en si la formación del futuro contador debe estar en el orden del saber contable o del hacer contable y cómo lograr una coherencia entre estos dos importantes campos de conocimiento, la cual permita que los profesionales den respuestas teóricas y prácticas a las nuevas problemáticas y retos de la profesión, atendiendo asertivamente las nuevas tendencias económicas, organizacionales y culturales que el mundo globalizado de hoy presenta.

\section{Desarrollo del pensamiento contable}

Conocer el desarrollo del pensamiento contable obliga realizar una breve revisión bibliográfica de las principales obras y exponentes de esta doctrina a lo largo de los años, desde el momento en el que se hablaba de contabilidad como un mecanismo de control usado por los comerciantes en el siglo $\mathrm{XV}$, hasta su actual definición construida a partir del desarrollo científico de la contabilidad a través de las revoluciones científicas, expresados en 
los paradigmas contables de Belkaoui, los programas de investigación expresados en términos contables por el profesor Cañibano y por último las tradiciones de investigación señaladas por Laudan.

\section{Historia de la contabilidad}

De acuerdo con la definición contenida en el Report of the Comittee on Accounting History de la American Accounting Association:

La historia de la contabilidad es el estudio de la evolución en el pensamiento contable, en sus prácticas, uso y ejercicio de las instituciones que se han creado en respuesta de los cambios del ambiente y de las necesidades sociales (Donoso, 2005, p. 21).

La historia se puede estudiar desde distintas ópticas, como su desarrollo legal y las instituciones, los documentos contables, el desarrollo de la contabilidad pública y privada o los textos y tratados contables desde el siglo XV; este último será el tema de interés o la fuente sobre la cual inicia este documento.

\section{Primeras publicaciones}

El desarrollo del comercio en la época del Renacimiento obligó a sus comerciantes a buscar una manera de conocer las compras, las ventas y los gastos, con el fin de evaluar si los resultados de su actividad generaban utilidades o pérdidas. Este tipo de control representa la partida simple y es el primer método conocido de representación contable que consiste en reconocer el hecho económico como una sola anotación.

En 1458, Benedetto Cotrugli escribió Della mercatura et del mercante perfetto con un aparte dedicado exclusivamente al uso de la partida doble, 
la cual representa un mismo hecho económico con dos anotaciones: una de origen y otra de destino, y propone el uso de tres libros: borrador, diario y mayor. Este texto fue inédito hasta el año 1573, razón por la cual no fue reconocido como el primero en hacer estas reflexiones y no se ha difundido su aporte a la historia de la contabilidad; sin embargo, desarrolla descripciones generales, utilidad y objeto en el uso de la partida doble. En 1494, se imprimió la obra de Fray Luca Bartolomeo de Pacioli, denominada Summa de Aritmética, Geometría, Proportioni et Proportionalita, y conocida como el primer libro de contabilidad, que incluye el capítulo "Tractatus XI Particularis de Computis et Scripturis", en el cual se explica el sistema de cuentas usado en Venecia. Especialmente, se describe el método de la partida doble para el registro de las transacciones económicas. Esta obra es considerada más amplia, profunda, sistemática y útil en cuanto a la parte práctica o uso de la partida doble.

En 1544, Domenico Manzoni publicó Quaderno doppio colsuo giornale, novamente composto, e diligentissimamente ordinato secondo ilcostume de Venetia, obra que retoma los elementos planteados por Paciolo en la Summa. No obstante, como el autor tenía conocimiento y experiencia en contabilidad, incluyó en el texto didácticas que ayudaron mucho a los lectores a tener un claro entendimiento del método y el registro.

Luego en 1590 apareció la primera obra de contabilidad escrita en español, denominada Libro de Caxa y Manual de cuentas de mercaderes y otras personas con la declaración dellos, escrita por Bartolomé Salvador de Solórzano. Según el maestro Hernández Esteve: "El propósito de estos textos fue el de suministrar a los comerciantes e interesados los conocimientos necesarios para que puedan llevar sus anotaciones contables por el sistema de partida doble" (Hernández, 1992, p. 19).

A partir del aporte de Paciolo y Cotrugli, con la partida doble muchos autores en Europa durante los siguientes siglos escribieron muchos textos, y aportaron así algunas novedades de tipo práctico en el uso de la partida 
doble como procedimiento contable. Dentro de los principales exponentes es importante hacer mención de los siguientes:

1. Francesco Villa Milán (1801-1884), divide en tres partes su pensamiento: conceptos económicos-administrativos, de la teneduría de libros y sus aplicaciones y organización administrativa, y rendición de cuentas.

2. Giuseppe Cerboni Elba (1827-1917), con su gran aporte de la "logismografía" como teoría y sistema de contabilidad que se refiere a la ordenación, razón de las cuentas.

3. Fabio Besta Tegli (1845-1922), conocido en Italia con el nombre de el Moderno Padre de la Contabilidad, le da una significación económica y de control a la contabilidad (CGN, 2011, p. 15).

\section{Corrientes del pensamiento contable más representativas}

A lo largo de los años, los diferentes autores mencionados anteriormente marcaron el inicio de las doctrinas de pensamiento contable, que a su vez se enriquecieron con la publicación de innumerables obras y aportes de orden teórico y técnico. Esta es otra manera de presentar la historia de la contabilidad.

\section{Doctrinas contistas y neocontistas}

Mileti, Berri, Gastaldi, Ilundain, Judais, Marcolin y Veron (2001, p. 69) consideran que "de 1494 a 1840 se señala como el período del contismo, las doctrinas contables se ocupan sólo del campo referido a la técnica de las anotaciones en partida doble" y su perfeccionamiento técnico se debe al crecimiento de la actividad comercial, el desarrollo de las operaciones de crédito y el nacimiento de las sociedades comerciales. 
Así, según Miletti

El neocontismo se preocupa por la noción del valor, captada y manifestada a través de las cuentas y de los balances, hay tres ramas principales:

1. El neocontismo continental con su Teoría de las dos series de cuentas.

2. El neocontismo norteamericano con sus Teorías descriptivas (hasta la década de los 60 en el siglo XX). En general la preocupación se centra en las cuentas y en los balances, en la explotación racional de los mecanismos de registración y en la utilización de los datos contables, como manifestación de un sistema de valores para dirigir adecuadamente la marcha económica de las explotaciones y resolver los conflictos de intereses entre los partícipes.

3. El neocontismo francés. Los autores ponen énfasis en el valor. Dumarchey y René Delaporte entre sus representantes (Mileti et al., 2001, p. 93).

\section{Doctrinas jurídico-personalistas}

Desplazan el objeto de la investigación contable de las cuentas y los registros a los derechos y obligaciones inherentes a un patrimonio objeto de administración (Mileti et al., 2001). Sus principales exponentes fueron Giuseppe Cerboni y Giovanni Rossi.

\section{Doctrinas de enfoque económico}

1. Controlismo: "El objeto de la contabilidad es el control económico de la riqueza hacendal” (CGN, 2011, p. 17).

2. Hacendalismo: definido como el sistema de personas y medios materiales e inmateriales que en sentido dinámico conducen a la sociedad a cumplir una finalidad (la familia, las empresas, las asociaciones, el Estado, todo es 
hacienda). Esta corriente terminó por no ofrecer un cuerpo de doctrina abarcante, competente para abarcar el campo contable (CGN, 2011, p. 17). Uno de sus exponentes fue Gino Zappa, el cual define a la Economía Hacendal como la "ciencia que estudia las condiciones de existencia y las manifestaciones de vida de las haciendas" (1937, p. 14), y distingue dentro de esta tres disciplinas: organización, técnica administrativa y contabilidad, a la que reduce al aspecto del registro (rilevazione) (Mileti et al., 2001).

3. Patrimonialismo: es la corriente contable con mayor repercusión en la actualidad y la cual considera la contabilidad como la ciencia que estudia los fenómenos del patrimonio hacendal. Su creador fue el italiano Vicenzo Massi (1927), quien fue alumno de Fabio Besta Massi. Esta doctrina divide los estudios contables en: estática patrimonial, dinámica patrimonial y revelación patrimonial (CGN, 2001, p. 17).

La contabilidad tuvo un importante desarrollo desde el siglo XV hasta el siglo XX, impulsada por la necesidad de medir los cambios económicos en las transacciones comerciales, la generación de valor percibida por los dueños de capital y la manera de controlar a los administradores de recursos. Estas problemáticas sociales fueron observadas y analizadas por los exponentes ya mencionados, los cuales centraron sus estudios en conceptos como el registro, la cuenta, el valor, el control y el patrimonio, entre otros. Durante aproximadamente 500 años se ofrecieron reglas sociales y económicas para el adecuado control de los recursos y la manera de representarlos; hecho que aumentó significativamente el saber contable en diálogo con las prácticas contables de la época y que transmitió este conocimiento de dos maneras: a través de textos escritos dirigidos a personas dedicadas a la contabilidad y a personas no expertas en el tema y de la enseñanza directamente a nuevos discípulos.

\section{Paradigmas contables}

El pensamiento contable a través de su historia ha construido conocimiento a partir de reflexiones sobre conceptos teóricos y prácticos que se han determinado como paradigmas — según la clasificación de Ahmed Belkaoui- 
o programas de investigación —-según Leandro Cañibano- debido a las características, intereses, métodos y otros elementos propios de esta forma de aproximación a la ciencia (Universidad Central, 2005).

Thomas S. Kuhn, en 1962, en su obra The Structure of Scientif Revolutions definió los paradigmas como las "Realizaciones científicas universalmente reconocidas que, durante cierto tiempo, proporcionan modelos de problemas y soluciones a una comunidad científica" (1971, p. 13). Su idea principal se denominó "revolución científica" y estuvo apoyado en el concepto de paradigma, entendido de dos maneras: "Por una parte significa toda la constelación de creencias, valores, técnicas, etc., que comparten los miembros de una comunidad dada. Por otra parte, denota una especie de elemento de tal constelación, las concretas soluciones de problemas que, empleadas como modelos o ejemplos, pueden remplazar reglas explícitas como base de la solución de los restantes problemas de la ciencia normal" (Gómez, 2004, p. 36, citando a Pheby, 1988, p. 37).

Primero significaría un éxito que se considera tan importante capaz de atraer a un grupo de científicos que ejercen una actividad en competencia; segundo, se entiende como un paso en el desarrollo científico apto para dar solución a problemas no resueltos. Los paradigmas se entienden como las teorías, las imágenes del mundo, las técnicas y métodos que comparte un grupo específico de investigación y los cuales ha formado desde las mismas bases epistemológicas y conceptuales. Cada paradigma o visión del mundo es aceptada por la comunidad científica, mientras llega un nuevo conocimiento capaz de cuestionar el paradigma, lo que trae como consecuencia ampliar el conocimiento en términos de las teorías o su rechazo total.

Sin embargo, el concepto kuhniano ha sido criticado por varios autores al acusarlo de ambiguo y vago, pero a la vez se ha usado con distintas finalidades. Rebaza (citando a Forget, 1998) señala que "un paradigma (i) no solo constituye un modelo empleado por un grupo social para solucionar 
problemas juzgados significativos, sino que, además (ii) puede ser adoptado como criterio normativo para juzgar el valor de un problema, de una metodología, de un vocabulario, de una teoría" (2007, p. 45).

Independientemente de la crítica que se haga al concepto, distintas disciplinas a lo largo de los años, han aumentado su acervo de conocimiento, han construido saber gracias a la identificación de sus propios paradigmas.

El concepto de paradigma en el orden de la disciplina contable fue abordado por primera vez en 1977 por la AAA (American Accounting Asociation), y fue revisado en 1981 por Ahmed Riahi Belkaoui en su obra Accounting theory, en la cual reconoce la existencia de los siguientes paradigmas contables básicos.

\section{Paradigma antropológico inductivo}

Este paradigma

Radica principalmente en las prácticas contables existentes y las actitudes gerenciales y administrativas hacia tales prácticas, el objetivo de investigación radica en lograr entender, explicar, y predecir las prácticas contables existentes, se identifican dos clases de teorías en este paradigma: 1. las que intenta explicar y justificar las practicas existentes y 2 . El papel de la gerencia en la determinación de técnicas contables e incluye la hipótesis de la nivelación de ingreso y los inicios de la teoría contable positiva (Montes, Soto y Valencia, 2006, p. 49).

De tal forma que las técnicas más comunes que se usan dentro de este enfoque son la observación, el razonamiento analítico y las técnicas empíricas. 


\section{Paradigma deductivo-beneficio verdadero}

Dentro de los autores que están inscritos en este paradigma encontramos a Alexander, Edwards, Bell, MacNeal, Moonizt, Paton, Sprouse y Moonizt y Sweeney. Estos teóricos comparten el interés por el enfoque deductivonormativo hacia la construcción de una teoría contable y la hipótesis de que la información de precios es más útil que la información convencional del costo histórico, al tomar decisiones de tipo económico (Montes et al., 2006). Este paradigma considera las siguientes orientaciones contables como método de valuación: nivel de precios, el principio de costo de sustitución, el principio de costo de escasez y valor presente.

\section{Paradigma utilidad-decisión}

Actualmente es el paradigma más influyente, dado que responde teóricamente a las necesidades actuales de los usuarios de la información financiera. Entre los autores representativos de este enfoque encontramos a Beaver, Kenelly y Voss y Sterling. Desde este paradigma se han desarrollado las siguientes tendencias de pensamiento.

\section{El modelo de decisión}

El sistema contable por sí solo no proporciona la información necesaria para la construcciones de modelos de decisión, pero sí debe suministrar la información relevante para la construcción de modelos de decisión de tipo económico, además de considerar los distintos eventos económicos que puedan afectar el principio de negocio en marcha. "El método general que se utiliza en el paradigma en cuestión es el análisis discriminatorio para clasificar varias de las agrupaciones a priori, depende de las características financieras individuales de la empresa" (Montes et al., 2006, p. 192). 
Este modelo de decisión se relaciona con los modelos PERT (Program Evaluation and Review Technic), programación lineal (máximos y mínimos), presupuestos de capital, compras y leasing.

\section{Comportamiento agregado del mercado}

El tema central de esta tendencia es la respuesta del mercado agregado a las variables contables; en otras palabras, el sistema contable produce información influenciada por el mercado. La teoría que sustenta el enfoque del comportamiento agregado es la hipótesis de eficiencia del mercado, que considera que la información empresarial ofertada se ve reflejada en los precios de las acciones, y que los precios de los títulos valores no están sesgados y reaccionan ante la presencia de nueva información (Montes et al., 2006).

\section{Usuario individual}

Este enfoque busca "la respuesta que el usuario individual presenta a las variables contables, se relaciona con el comportamiento humano y cómo lo afectan las variables contables" (Montes et al., 2006, p. 193). Entre las técnicas usadas encontramos la observación, las entrevistas, los cuestionarios y la experimentación.

\section{Paradigma economía-información}

Los principales temas de estudio de este paradigma son 1) la información es una comodidad económica y 2) la obtención de información representa un problema de escogencia económica. Se entiende la información como la materia prima necesaria para tomar decisiones; se abordan las teorías de los equipos, de la decisión estadística y de la teoría económica de la selección, se hace un análisis normativo de las alternativas de acción del usuario de la información (Montes et al., 2006). 
En el sentido estricto, los diversos enfoques presentados denominados paradigmas dan cuenta de la gran senda por la cual ha transitado el pensamiento contable, es conocimiento acumulativo, útil para las distintas demandas o necesidades sociales que se identifiquen en un momento histórico.

Los conceptos prácticas contables y teorías contables son el objeto de estudio de esta investigación. Vistos desde la perspectiva de la práctica docente, dos de los principales paradigmas contables, el paradigma antropológicoinductivo y deductivo beneficio verdadero, se centran en estos conceptos como los principales temas de estudio. El pensamiento contable se ha construido a partir de reconocer prácticas que se sustentan en teorías y en nuevas maneras de haceres o técnicas o nuevas prácticas que promuevan un nuevo saber que remplace al anterior. No es posible concebir en la disciplina contable una práctica sin teoría y una teoría que no se sustente en una necesidad de tipo práctico, lo cual se demuestra en los textos desarrollados por los exponentes de estos paradigmas.

\section{Programas de investigación contables}

El término programa de investigación fue usado por Imre Lakatos (1993), autor que plantea que el conocimiento se construye a partir de los programas de investigación y no de paradigmas, como lo planteado por Kuhn. Cada programa está compuesto por varias teorías y estas se conforman de manera continua con la anterior y no por medio de remplazo.

En cuanto a los programas de investigación, Cañibano Calvo (1996) señala, citando a Lakatos (1993), que un programa de investigación consiste en reglas heurísticas, unas de carácter negativo por cuanto nos dicen qué caminos de investigación hay que evitar, y otras positivas, toda vez que indican los caminos que hay que seguir. También se puede entender por programa de investigación científica "una configuración de teorías interconectadas, ninguna de las cuales se considera totalmente autónoma 
por lo que es difícil descartar teorías individuales sin hacer referencia al programa de investigación como un todo" (Gómez, 2004, p. 23).

En contabilidad, el profesor Cañibano (1996) propone tres programas de investigación. El primero es el legalista, que responde a las reglas y a la función de registro, y el cual inicia con la aparición de la partida doble. El objetivo principal de este es de suministrar información sobre la situación del propietario (Mileti et al., 2001). Paralelamente, esta información sirve de garantía a terceros a partir de su estructura legal y de herramienta para el usuario en la toma de decisiones económicas. Claramente, la orientación legalista (jurídica) de la contabilidad era jerárquicamente superior a las pretensiones económicas (Mejía, Montes y Echeverri et al., 2006).

El segundo programa es el económico, que está condicionado a la realidad económica y empresarial. Aquí se intenta la búsqueda y registro de una verdad única, el cálculo del beneficio y de la situación patrimonial, sin importar quién la recibe y por qué. Esta etapa buscó e incorporó conceptos basados en la teoría económica y en la microeconomía, en una búsqueda de "la verdad" que facilitara el cálculo y la eficiente distribución de los recursos. En esta etapa existe una preponderancia de la regulación por sobre la innovación, realizándose investigaciones a priori (Mileti et al., 2001). Así,

la finalidad de la información contable queda estrechamente vinculada al conocimiento de la realidad económica, tanto del orden nacional como empresarial. Los objetivos de la información contable, sin abandonar los estrictamente legales, quedan más bien orientados hacia aspectos puramente económicos de la actividad empresarial (Cañibano, 1996).

El tercer programa busca rigurosamente entender los fenómenos contables. El artículo titulado "Hacia una Fundamentación General y Axiomática de la Contabilidad", de Richard Mattessich (1957), constituye un punto de partida para el programa de investigación formalizado. Mattessich presenta una estructura formalizada de la contabilidad a través de axiomas, definiciones, teoremas y requisitos. Se apoya en el álgebra matricial y hace una 
presentación formal de la contabilidad. En el artículo prima su esquema semántico sobre la intencionalidad simbólico-matemática.

Por otra parte, la investigación contable logró una estructura formalizada solo hasta 1964, y en ese año el mismo autor publicó Contabilidad y métodos analíticos, (traducida al español en 2002, Argentina). En este texto formaliza la contabilidad con la ayuda de la teoría de conjuntos, "la rigurosidad formal permite un análisis de gran profundidad de aspectos de la mayor relevancia, tales como medición, valoración y predicción” (Mejía et al., 2006, p. 59).

Cada uno de estos enfoques tiende a transformarse con el paso de los años, lo que da origen a la necesidad de atender las nuevas tendencias que presenta el vertiginoso mundo de los negocios y la manera de estructurarlos, así como a repensar nuevas tradiciones de investigación.

La comunidad de investigadores contables reconoce la vigencia de los tres programas mencionados: el legalista, el económico y el formalizado. Los temas centrales de estudio de los investigadores en contabilidad han estado vigentes desde el siglo XV hasta la actualidad, con un importante desarrollo interdisciplinar.

En el programa legalista la ciencia jurídica se desarrolló a la par con la disciplina contable (las leyes regulan las prácticas contables). Asimismo, dicho programa pone en evidencia la pertinencia de reconocer un marco conceptual y unos principios contables generales que fundamentan el reconocimiento por parte de los profesionales de los hechos económicos y las consideraciones por observar en la elaboración de la información contable. En otras palabras, la práctica se guía por la teoría.

En el segundo programa, se estudia cómo la realidad económica es el primer fenómeno que se presenta par ser investigado y reconocido por una teoría contable y posteriormente aplicado. Por ejemplo, la necesidad de un cálculo de beneficio verdadero que permita tomar adecuadas decisiones de inversión por parte de los distintos usuarios. El término verdadero espera 
que las cifras contables reflejen la realidad económica y no una realidad histórica. Así pues, el problema central teórico y práctico en este programa es la validación del método de valoración que se debe dar a los recursos.

En el tercer programa - el formalizado - se hace un riguroso uso de la lógica matemática para justificar el carácter científico de la contabilidad. De igual forma, este programa propone unas hipótesis básicas que permitan a los investigadores contables usarlas como ciertas para la construcción de la misma ciencia y para el hacer contable. Las reglas propuestas definen el uso de instrumentos y herramientas que apoyan la elaboración de los informes.

Estos programas ponen en evidencian que a partir de observar y analizar una realidad económica dinámica y cambiante, se propone una interpretación contable que se traduce en un aplicación práctica, que produce otras formas de interpretar y representar esos hechos económicos y la producción de información contable útil para los distintos usuarios.

\section{Tradiciones de investigación en contabilidad}

Richard Matessich, retomando a Laudan (1977), ha sido uno de los autores contemporáneos más destacados en la comunidad contable. Él se puso en la tarea de reinterpretar los paradigmas señalados por Belkaoui (2004) en redes teóricas o tradiciones investigativas, y fue quien definió a la contabilidad como una ciencia aplicada, orientada a unos propósitos específicos. En este sentido, dicho autor propone las siguientes tres tradiciones o programas de investigación.

\section{Tradición o programa de gerencia o rendición de cuentas}

Se apoya en la teoría de la agencia, en la cual la contabilidad cumple con la función de control de los recursos del propietario o principal, sobre la gestión dada a estos recursos por el agente. Este programa está constituido por tres fases: 
La primera se denomina pro-puesta de periodización, en la cual "el monitoreo de la gerencia directiva puede ser mejor satisfecho con la apropiada asignación de costos y beneficios de inversión a un periodo específico" (Mattessich, 1987, p. 103). En esta parte se hace uso de unos principios contables, como el costo histórico, la asignación periódica de costos y beneficios, costos por depreciación y el supuesto de negocio en marcha.

En la segunda fase, denominada propuesta original de la gerencia, se señala el conflicto de intereses que se genera de la relación principal-agente, dado que en varias ocasiones los objetivos que persiguen uno u otro coinciden. Para solucionar el conflicto, se hace uso de un contrato que dirige a la consecución de los objetivos de ambas partes, sin asumir costos adicionales.

La tercera fase, llamada propuesta agencia-información, se centra en un contrato que celebran el principal y el agente, orientado a la minimización de riesgos del negocio debidamente informado. Acá se pueden tomar dos posiciones: la primera es que el contrato indique que el agente obtiene un salario fijo por su gestión, desde el control del principal, y los resultados positivos (beneficios) y riesgos serán asumidos por el principal. La segunda posición es que el principal reciba una renta derivada de la gestión de los recursos de este y el agente asuma el riesgo y obtenga los excedentes generados. Con esta opción, ambas partes participan de los riesgos y de los beneficios.

\section{Tradición o programa de valuación-inversión}

Se considera con este enfoque que la contabilidad debe valuar económicamente los recursos y los derechos. El enfoque presenta tres propuestas o métodos de valuación. La primera hace referencia al valor presente o valor corriente, utiliza como recurso el valor actual contable, el valor de reposición y el valor de realización. La segunda propuesta hace referencia al riesgo compartido, basado en la teoría del portafolio, los riesgos de mercado y de los títulos valores. La última propuesta es la de mercado de capitales. 


\section{Tradición o programa de información-estrategia}

Denominada también como una contabilidad orientada a unos fines específicos. Se manifiesta en la distinción que hace Mattesich entre "la teoría básica no interpretada (abstracta) y las interpretaciones orientadas" (Mattessich, 1987, p. 109). Este es uno de los postulados principales de lo que hoy se conoce como la teoría general de la contabilidad y los sistemas contables.

El desarrollo del pensamiento contable se ha dado gracias a que algunos hombres se han detenido a construir saber contable a partir de pensar unos problemas que han cambiado a través de la historia, con el desarrollo económico, empresarial y social. Problemas que al tratar de abordarlos cubren algunas necesidades, pero nacen otras. La construcción de este pensamiento contable se ha dado gracias a los distintos intereses de investigación contables evidentes a lo largo del tiempo.

\section{La investigación contable}

La investigación es: "la búsqueda del saber que amplía las fronteras del conocimiento y de su aplicación, compartidas hasta ahora por las distintas comunidades científicas. Esta búsqueda se obtiene con procesos diferenciados y autónomos" (Campo y Restrepo, 2002, p. 83).

Se identifican dos grandes grupos que se ocupan de la investigación contable. El primero aborda problemáticas contables a nivel técnico, teórico y epistemológico, lo que ha permitido la construcción de un marco teórico referencial de las prácticas contables, denominada investigación disciplinar. El segundo centra su análisis en aspectos propios de la práctica profesional, su aplicación en aspectos específicos como la gestión, las finanzas, el control, lo fiscal, etc. Esta es la investigación de corte profesional (Barrios, Sánchez y Lemos, 2010). 


\section{La investigación de corte disciplinar}

Se preocupa por las condiciones internas lógicas de la estructura conceptual de la teoría contable, es decir, de la teoría general y de las teorías de sus aplicaciones, y de sus relaciones con otros campos del conocimiento como la economía, la administración, el derecho, la sociología, la filosofía (Gómez, 2003 p. 142),

al hacer uso de conceptos de otras disciplinas como las anteriormente mencionadas. Se valida y formaliza el corpus teórico contable a partir de las relaciones inter $\mathrm{y}$ transdisciplinarias.

La investigación profesional o aplicada aborda problemáticas inherentes a las organizaciones y sus necesidades de información contable, administrativa o de gestión, sea para controlar, tomar decisiones o para suplir otra necesidad inherente de la empresa. Es de corte instrumental y hace uso de conocimientos de orden jurídico, estadístico, metodológico, procedimental y tecnológico; además, construye determinados saberes que apoyan el hacer contable.

A partir de esta afirmación, se manifiesta la relación bidireccional, necesaria e irrompible, entre teoría contable y práctica contable, vínculo que desde los año ochenta ha sido discutido en términos de si la investigación dinamiza la práctica contable y sus aportes han sido significativos para la disciplina contable. Algunos autores afirman que la investigación es un proceso que se dimensiona como una solución a la práctica. También se ha afirmado que la investigación es promovida por los académicos contables, como una respuesta requerida por los profesionales; igualmente, algunos teóricos la definen como ese intento decidido por mejorar la realidad o las prácticas profesionales de estas discusiones.

En este sentido, se identifican dos grupos: los profesionales y los académicos investigadores, los cuales han perseguido intereses distintos. Los primeros centran su atención en los métodos, procedimientos, aportes de la 
tecnologías, el aspecto legal y su incidencia en la práctica profesional contable en las organizaciones, y los segundos en el pensar la ciencia contable y a través de la investigación y los discursos enriquecer el corpus epistemológico que den a la contabilidad su estatus de ciencia. Sin embargo, a pesar de las innumerables investigaciones, artículos y discusiones a nivel mundial y regional, no se ha logrado construir canales de comunicación entre estos dos grupos. Los profesionales han expresado fuertes críticas respecto al bajo nivel de utilidad de las investigaciones realizadas y el bajo impacto de su promoción en el medio profesional. Por parte de los académicos, las críticas se han dirigido especialmente a expresar que las comunidades de profesionales contables solo esperan investigaciones que respondan de manera inmediata a la práctica. Una solución a esta problemática podría ser lograr dar lectura de las demandas que surgen en la práctica e investigar cómo se pueden solucionar las mismas, no respondiendo únicamente a esas necesidades prácticas, sino a la par, y pretendiendo dar aportes teóricos que enriquezcan la disciplina.

\section{Tendencias actuales de la investigación en contabilidad}

La construcción del conocimiento contable ha sido un continuo proceso crítico que, respondiendo a las necesidades y realidades socio económicas, ha estructurado saberes contables que han apoyado a los distintos actores en su imagen instrumental como herramienta de control y gestión entre los comerciantes en el siglo XV y en las revisiones conceptuales como los principios, las doctrinas y postulados que guían el ejercicio profesional a nivel regional y mundial.

La literatura contable identifica tres grandes tendencias de la investigación en contabilidad a nivel mundial. La primera, denominada el enfoque de modelo-decisión centrado en la información necesaria para la toma de decisiones, "se concentra en determinar qué información es utilizada o adecuada al uso para determinadas decisiones" (Díaz y Riadi, 1999, p. 13). La segunda tendencia que se identifica es la investigación en mercado de capitales o teoría de la eficiencia del mercado. Esta tendencia, "A partir de investigaciones empíricas 
(deductivas), que muestran las reacciones de los precios de las acciones sobre la base de la hipótesis de mercados eficientes, asume gran importancia en la revelación contable" (Díaz y Riadi, 1999, p. 14). El comportamiento del mercado puede ser un indicador válido para seleccionar entre varias alternativas contables, a causa del impacto de cada una de esas alternativas en él.

Se está en consecuencia según Tua "Ante una variante de los estudios de capacidad predictiva de la información financiera aplicada a los mercados de capitales, en los que los estados financieros, a través del mecanismo de formación de precios, contribuyen a la distribución óptima de la riqueza” (Tua Pereda, 1983, p. 967).

La tercera tendencia es denominada la teoría de agencia, definida

como un contrato en el que una o más personas - el principal- encarga a otra persona -el agente- el desarrollo de un servicio en su nombre, lo que supone delegar parte de la toma de decisiones en el agente. El vínculo contable con la teoría de agencia, se traduce entre otros, en la selección que los directivos podrían hacer, sobre alternativas contables, a objeto de maximizar la medición de los resultados, si su propio beneficio se ve con ello incrementado (Aquel, Cicerchia y Mileti, 2010, p. 21).

La teoría encontrada en la literatura contable en su gran mayoría responde a artículos derivados de proyectos de investigación que trabajan alguna de las tres tendencias mencionadas o trabajos independientes que también se centran en estos contenidos. Todos estos documentos hacen parte de lo que hoy se denomina teoría contable, la cual observa el mundo real e intenta solucionar problemas prácticos, entre otras de sus bondades.

\section{Saber contable y hacer contable}

La dicotomía entre teoría y práctica es posible plantearla en términos del profesor Záa (2010, p. 53) (hacer contable y pensar contable), el cual plantea que 
para el desarrollo de la primera dimensión, el Hacer Contable, requiere conocimientos matemáticos, estadísticos, metodológicos y procedimentales; y para la segunda, el Pensar Contable, es necesario el manejo amplio, profundo y riguroso del lenguaje de la contabilidad, sus métodos científicos, los niveles de lógica de esta disciplina, los aportes de otras ciencias, el cuerpo argumental, y en fin, el estatuto científico contable, para poder interpretar, comprender y explicar los objetos de los cuales se ocupa la contabilidad.

El saber contable no implica una definición formal o descripción concreta de alguna práctica, es la simplificación de la realidad en modelos conceptuales que explican, comprenden, interpretan, interrelacionan y construyen los fenómenos naturales y los hechos sociales por medio de relaciones causales o interrelaciones y motivadores de la acción social (Fried Schnitman, 2002, citado en Gómez, 2003). En contabilidad, el saber contable no es simplemente normas o los principios contables.

Por su parte, la práctica no es simplemente el desarrollo de un conjunto de técnicas de registro. La práctica no es la partida doble o la cuenta, el soporte, el registro, el proceso o, incluso, la interpretación del dato y la información. El hacer contable es la transformación intencionada del mundo a partir de un modelo de comprensión del mismo. La práctica es hacer el mundo a imagen y semejanza del modelo teórico. Dado que las teorías pueden ser erróneas o inadecuadas, el espíritu científico que construye teorías debe ser crítico. Debe constantemente criticar las teorías y contrastarlas con la realidad factual por medio de la praxis. También los hechos pueden desbordar las formas de entendimiento teórico sobre ellos, por eso la reflexión debe ser aguda y permanente (Gómez, 2006).

Se debe definir lo que se va a entender en este documento como saber contable, dadas las múltiples concepciones de esta categoría, situación derivada de las variadas escuelas, doctrinas y corrientes de pensamiento contable. Así, para Hendricksen (1965) (citado en Gómez, 2006) la teoría o saber contable de la contabilidad se entenderá como el conjunto de principios hipotéticos, conceptuales y pragmáticos que forman el marco general 
de referencia mediante el cual se puede evaluar la práctica de la contabilidad y guiar el desarrollo de nuevas y buenas prácticas y procedimientos.

Gómez (citada en Macintosh y Hopper, 2005), que habla en términos de teoría contable, la define como el corpus ontológico, epistemológico y metodológico que pretende explicar-comprender, de forma sistemática y racional, las prácticas, procesos, procedimientos, técnicas, tecnologías e incluso las reglas contables. Algunas teorías trascienden el espíritu descriptivo, abstracto, universalista y funcional, para relacionar el rol contable con su entorno social, cultural, institucional y político" (p. 4).

$\mathrm{Al}$ intentar interpretar las anteriores definiciones del concepto se identifican unos elementos comunes que permiten definir el saber contable como la interpretación de una realidad económica financiera, que facilita la construcción de dispositivos o herramientas que aporten a la solución de los problemas que subyacen de esta realidad. De este modo, uno de los objetos de estudio del saber contable es el hacer contable y es una construcción derivada de serios procesos de investigación, cuyo origen son las problemáticas que se evidencian en la realidad social, específicamente en las dimensiones administrativas, financieras, económicas y hoy también la ambiental.

Con el fin de lograr una dinámica equilibrada entre el hacer y el pensar de los futuros contadores públicos, es necesario producir cambios en el aula a partir de la interpretación, comprensión y reflexión de las categorías de la epistemología contable, soporte del hacer contable y el repensar diversos objetos de estudio de la contabilidad que aporten a lo profesional y disciplinar.

En palabras de Burbano:

la contabilidad como fruto de la acción humana y de los hechos sociales, es uno de los elementos esenciales para motivar, impulsar y determinar nuevas acciones y nuevos hechos particularmente los de producción y distribución de riqueza, entre otros (1989, p. 32). 
Por tanto, otorgar la importancia debida al cuerpo teórico y reconocer a la contabilidad como ciencia histórica social, que responde a las necesidades del hombre en su contexto económico, financiero y administrativo, permite al ser-contable, que el pensamiento no se simplifique en el orden de lo técnico. Se requiere entonces un cambio de actitud, una conversión intelectual que lleve a superar el carácter empírico de la contabilidad a partir de la adecuada reflexión de la teoría.

El profesional que apropia el saber contable estará en capacidad de ir más allá de la descripción y la explicación de los fenómenos económicos, y su trabajo se centrará en la interpretación, el análisis, la comprensión, la crítica y la innovación del mundo económico donde se desenvuelva (Gómez, 2003). Lo principal para este tipo de Contador es entender el fenómeno, sus implicaciones micro y macroeconómicas, comprender las razones del Estado por normalizar de cierta manera los asuntos derivados del fenómeno objeto de estudio, para así finalmente - y de manera responsable - informar a la sociedad los resultados y generación de riqueza por parte de las organizaciones. La búsqueda del saber será un proceso continuo y permanente a lo largo de su trayectoria y ante cualquier situación, incertidumbre o decisión el porqué y para qué será el punto de partida, independientemente de la limitación normativa o tecnológica.

\section{Conclusiones}

El saber contable implica conocer las corrientes de pensamiento contable, las implicaciones de las doctrinas, paradigmas y tendencias investigativas; entender cómo este cuerpo teórico da respuesta a las necesidades de orden práctico que se han presentado en las distintas culturas, estar en capacidad de dar lectura de estas y reinterpretarlas y usarlas para dar solución a nuevas problemáticas contables que se presentan en el mundo organizacional o académico; también debe comprenderse la importancia de la teórica contable. Reconocer que el uso adecuado de las técnicas, los registros, la tecnología, los conceptos de otras disciplinas como la Estadística y la 
Matemática Financiera, es propio de un excelente e íntegro profesional de la Contaduría Pública en el país, y es el perfil contable que demandan hoy las organizaciones mundiales.

\section{Referencias}

Aquel, S.; Cicerchia, L. y Mileti, M. (2010). Perspectivas contemporáneas de investigación en Contabilidad. Trabajo presentado en las XVI Jornadas de Epistemología de las Ciencias Económicas. Facultad de Ciencias Económicas. Buenos Aires, Argentina. Recuperado de http://www.econ.uba.ar/www/ institutos/epistemologia/marco_archivos/trabajos_XV_archivos/Aquel,\%20 Cicerchia\%20y\%20Mileti-\%20PERSPECTIVAS\%20CONTEMPOR.pdf

Barrios, C., Fúquene, S. y Lemos, J. (2010). Desarrollo de la investigación contable en el Centro Colombiano de Investigación Contable. Grupo de Investigación Pensamiento y Prácticas Investigativas y Prácticas Contables. Pontificia Universidad Javeriana, Cali.

Belkaoui, A. (2004). Accounting Theory. Boston: Cengage Learning, EMEA.

Burbano, J. E. (1989). Contabilidad: Análisis Histórico de su Objeto y Método. Revista Pliegos Administrativos y Financieros, 12, 89-104.

Cañibano, L. y Angulo, J. A. (septiembre, 1996). Los Programas de Investigación en Contabilidad. Revista Universidad de Antioquia, 29, 13-61.

Colombia, Contaduría General de la Nación (2011). La contabilidad y su utilidad en el sector público. Recuperado de http://www.contaduria.gov.co/wps/wcm/ connect/ec735a17-2fa5-4cfa-b9c8-848e6f1dc287/La_contabilidad_y_su_utilidad.pdf?MOD=AJPERES

Díaz, J. y Riadi, F. (1999). Contabilidad y Ciencia. Revisión y Propuesta Desde Una Perspectiva Académica. Revista Capic, 1, p.1-23.

Donoso, R. (2005). Estado Actual de la Investigación en Historia de la Contabilidad. Revista de Contabilidad, 8(15), 19-44.

Gómez, M. (2003, julio-diciembre). Algunos comentarios sobre la potencialidad de la investigación en contabilidad. Innovar. Revista de Ciencias Administrativas y Sociales, 21, 139-144. 
Gómez, M. (2006). Comentarios sobre el aprendizaje construcción de la teoría contable. Lúmina, 7, 129-153.

Gómez, R. (2004). Evolución científica y metodológica de la Economía. Recuperado de http://www.eumed.net/cursecon/libreria/rgl-evol/rgl-metod.pdf

Hernández, E. (1992). Benedetto Cotrugli, precursor de Pacioli en la exposición de la partida doble. Cuadernos de Estudios Empresariales, 2, 87-99.

Kuhn, T. (1971) [1962]. La estructura de las revoluciones científicas. México D.F.: Fondo de Cultura Económica.

Lakatos, I. (1993). La metodología de los Programas de investigación científica. Madrid: Alianza.

Laudan, L. (1977). El progreso y sus problemas: hacia una teoría del crecimiento científico. Madrid: Ediciones Encuentro, S.A.

Mattessich, R. (2002). Contabilidad y métodos analíticos: medición y proyección del ingreso y la riqueza en la microeconomía y en la macroeconomía. Buenos Aires: La Ley.

Mejía, E., Montes, C. y Echeverri, D. (2006). Programas de investigación en contabilidad. Bogotá: Universidad Libre de Colombia.

Marcolini, S. y Veron, C. (2001). Evolución histórica de la contabilidad y su relación con la investigación y regulación contable en Estados Unidos, Sur de Europa y Argentina. Sextas Jornadas Investigaciones en la Facultad de Ciencias Económicas y Estadística. Instituto de Investigaciones Teóricas y Aplicadas Escuela de Contabilidad. Recuperado de www.fcecon.unr.edu.ar

Montes, C.; Mejía, E. y Salazar, J. (2006). Paradigmas en contabilidad. Cali: Artes Gráficas del Valle.

Rebaza, I. (2007). Fundamentos para el Desarrollo de una Investigación Contable. Lima: Fondo Editorial del Colegio de Contadores Públicos de Lima. Restrepo, M. y Campo, R. (2002). La docencia como práctica, el concepto, un estilo, un modelo. Bogotá: Pontificia Universidad Javeriana.

Tua Pereda, J. (1983, octubre). Subprogramas de investigación en la emisión de principios contables. Revista Técnica Contable, 35, 361-380.

Universidad Central (2005). Proyecto Académico de Carrera. Bogotá: Universidad Central.

Zaá, J. (2010). Pensar la ciencia contable. Compilación de artículos propios [en prensa] Caracas: Federación de Colegios de Contadores Públicos de Venezuela. 